\title{
Correction to: A Course in Multivariable Calculus and Analysis
}

\section{Correction to:}

S. R. Ghorpade, B. V. Limaye, A Course in Multivariable

Calculus and Analysis, Undergraduate Texts in Mathematics, https://doi.org/10.1007/978-1-4419-1621-1

After initial publication of the book, various errors were identified that needed correction. All corrections listed below have been updated within the current version. Note that the correction to Chapter 7 starting p. 422 changes the pagination of all subsequent material, starting from "Absolute Convergence and Conditional Convergence", previously p. 425, now p. 427.

In the following, $\mathbf{p} . \mathbf{i},+\mathbf{j}$ means the $j$ th line from the top on page $i$, whereas $\mathbf{p} . \mathbf{i},-\mathbf{j}$ means the $j$ th line from the bottom on page $i$. Here page $i$ refers to the $i$ th page in the original edition of the book published in 2010. The text to be changed and the corresponding corrected version usually appear in quotes.

\section{Chapter 1}

p. 5, $+\mathbf{1 6}$ : Change "least upper bound" to "greatest lower bound"

p. 10, -12: Change " $f(x, y) \geq 0$ " to " $f(x, y)>0$ "

p. 34, -6: Change "handled them with" to "handled with" \footnotetext{
https://doi.org/10.1007/978-1-4419-1621-1_1 https://doi.org/10.1007/978-1-4419-1621-1_2 https://doi.org/10.1007/978-1-4419-1621-1_3 https://doi.org/10.1007/978-1-4419-1621-1_4 https://doi.org/10.1007/978-1-4419-1621-1_5 https://doi.org/10.1007/978-1-4419-1621-1_6 https://doi.org/10.1007/978-1-4419-1621-1_7 https://doi.org/10.1007/978-1-4419-1621-1
}

The updated online version for these chapters can be found at 


\section{Chapter 2}

p. 45, +2: Change "If" to "Let $a_{n} \rightarrow a$. If"

\section{Chapter 3}

p. 88, +5: Change " $x_{0} \in[c, d]$ " to " $x_{0} \in[a, b]$ "

p. 97, -13: Change " $u: D \rightarrow \mathbb{R}^{2}$ are" to " $u: D \rightarrow \mathbb{R}$ are"

p. 97, -8: Change " $f$ " to " $g$ "

p. 118, -10: Change " $f(x, y)$ " to " $f\left(x_{1}, y_{1}\right)$ "

p. 118, -10: Change at two places " $k \frac{\partial}{\partial x}$ " to " $k \frac{\partial}{\partial y}$ "

p. 154, + 9: Change " $\sqrt{h^{2}+k^{2}} "$ to " $|h|+|k| "$

p. 156, -2: Change " $(y-b)(z-c)$ " to " $(y-c)(z-p)$ "

\section{Chapter 4}

p. 166, +22: Change " $\mu h(x, y, z)$ " to " $\mu \nabla h(x, y, z)$ "

\section{Chapter 5}

p. 213, +11: Change " $R$ " to " $\mathbb{R}$ "

p. 213, -6: Change " 5.19 " to " 5.23 "

p. 219, -6: Change " $0 \leq a<b$ and $0 \leq c<d$ " to " $0<a<b$ and $0<c<d^{\prime \prime}$

p. 220, - 13: Change "Moreover, by Proposition 5.28, we have" to "Moreover,"

p. 224, +3: Change " $[a, b) \times[c, d)$ " to " $[a, b] \times[c, d]$ "

p. 238, -19: Change "iterated integral" to "integral"

p. 245, +14: Change "and $D_{1} \cap D_{2}$ are" to "and $D_{1} \cap D_{2}$ is"

p. 268, +18: Change "Exercise 43" to "Exercise 43 of Chapter 3"

p. 272, -10: Change " $d(y, z)$ " to " $d(x, y)$ "

p. 272, -9: Change " $f(x, y, z)$ " to " $\int_{\phi_{1}(x, y)}^{\phi_{2}(x, y)} f(x, y, z) d z$ "

p. 275, +16: Change " $f$ " to " $f \circ \Phi$ "

p. 278: Change Figure 5.26 to the revised figure provided below.

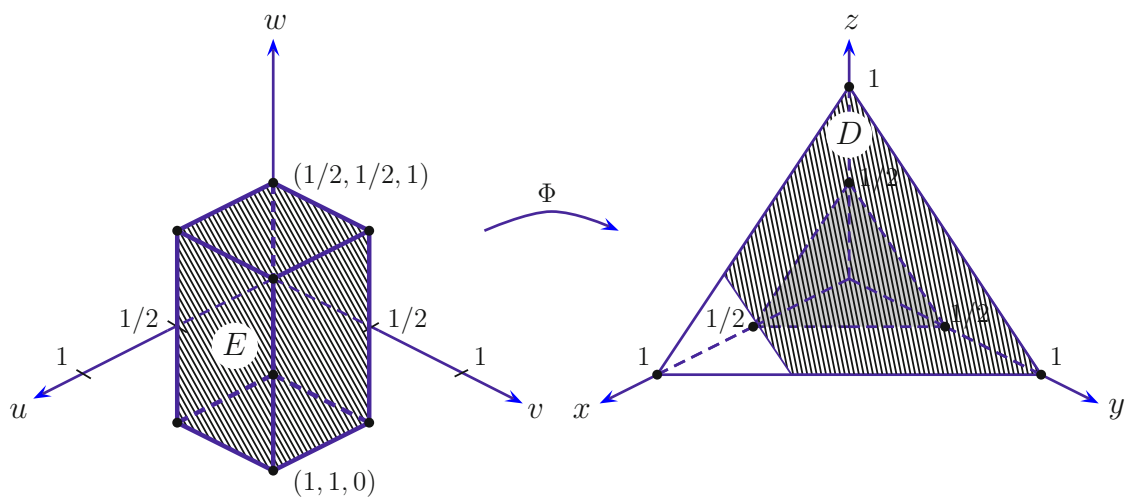


p. 282, -3: Change " $x \leq y$ " to " $y \leq x$ "

p. 285, +19: Change " $49 / 192 "$ to " $49 / 576$ "

\section{Chapter 6}

p. 302, -16: Change " $D, y z$-plane" to " $D$ by the $y z$-plane"

p. $303,+11$ : Change " $[-\pi, \pi] \times\left[f_{1}(x), f_{2}(x)\right] "$ to " $\left[f_{1}(x), f_{2}(x)\right] \times[-\pi, \pi] "$

\section{Chapter 7}

p. $422,-6$ to p. $425,-11$ : Change the entire text from the statement of the Integral Test until the beginning of the next subsection to the revised text provided below.

Proposition 7.57 (Integral Test). Let $f:[1, \infty) \times[1, \infty) \rightarrow \mathbb{R}$ be a nonnegative monotonically decreasing function. If $\iint_{[1, \infty) \times[1, \infty)} f(s, t) d(s, t)$ is convergent, then the double series $\sum \sum_{(k, \ell) \geq(2,2)} f(k, \ell)$ is convergent, and

$$
\sum_{k=2}^{\infty} \sum_{\ell=2}^{\infty} f(k, \ell) \leq \iint_{[1, \infty) \times[1, \infty)} f(s, t) d(s, t) .
$$

Conversely, if the full double series $\sum \sum_{(k, \ell) \geq(1,1)} f(k, \ell)$ is convergent, then the improper double integral $\iint_{[1, \infty) \times[1, \infty)} f(s, t) d(s, t)$ is convergent, and

$$
\iint_{[1, \infty) \times[1, \infty)} f(s, t) d(s, t) \leq \sum_{k=1}^{\infty} \sum_{\ell=1}^{\infty} f(k, \ell)
$$

and moreover, the improper integrals $\int_{[1, \infty)} f(s, 1) d s$ and $\int_{[1, \infty)} f(1, t) d t$ are convergent, and

$\sum_{k=1}^{\infty} \sum_{\ell=1}^{\infty} f(k, \ell) \leq f(1,1)+\int_{1}^{\infty} f(s, 1) d s+\int_{1}^{\infty} f(1, t) d t+\iint_{[1, \infty) \times[1, \infty)} f(s, t) d(s, t)$.

On the other hand, if the improper double integral $\iint_{[1, \infty) \times[1, \infty)} f(s, t) d(s, t)$ diverges to $\infty$, then the full double series $\sum \sum_{(k, \ell) \geq(1,1)} f(k, \ell)$ diverges to $\infty$, whereas if the double series $\sum \sum_{(k, \ell) \geq(2,2)} f(k, \ell)$ diverges to $\infty$, then the improper double integral $\iint_{[1, \infty) \times[1, \infty)} f(s, t) d(s, t)$ diverges to $\infty$.

Proof. Since $f$ is monotonic, by part (i) of Proposition 5.12, $f$ is integrable on $[1, x] \times[1, y]$ for every $(x, y) \geq(1,1)$. Define $F:[1, \infty) \times[1, \infty) \rightarrow \mathbb{R}$ by $F(x, y):=\iint_{[1, x] \times[1, y]} f(s, t) d(s, t)$. Since $f$ is nonnegative, by Corollary 5.10, 
the function $F$ is monotonically increasing. Hence Proposition 7.55 implies that the improper double integral $\iint_{[1, \infty) \times[1, \infty)} f(s, t) d(s, t)$ is convergent if and only if the set $\left\{F(m, n):(m, n) \in \mathbb{N}^{2}\right\}$ is bounded above, and in this case

$$
\begin{aligned}
\iint_{[1, \infty) \times[1, \infty)} f(s, t) d(s, t) & =\sup \{F(x, y):(x, y) \in[1, \infty) \times[1, \infty)\} \\
& =\sup \left\{F(m, n):(m, n) \in \mathbb{N}^{2}\right\} \\
& =\lim _{(m, n) \rightarrow(\infty, \infty)} F(m, n) .
\end{aligned}
$$

Here the penultimate equality follows since $F$ is monotonically increasing, and the last equality follows from part (i) of Proposition 7.4. Similarly,

$$
\iint_{[1, \infty) \times[1, \infty)} f(s, t) d(s, t) \text { diverges to } \infty \Longleftrightarrow F(m, n) \rightarrow \infty \text { as } m, n \rightarrow \infty .
$$

Now let us define

$$
a_{k, \ell}:=\iint_{[k, k+1] \times[\ell, \ell+1]} f(s, t) d(s, t) \quad \text { for }(k, \ell) \in \mathbb{N}^{2}
$$

and

$$
A_{m, n}:=\sum_{k=1}^{m} \sum_{\ell=1}^{n} a_{k, \ell} \quad \text { for }(m, n) \in \mathbb{N}^{2} .
$$

By the Domain Additivity of Double Integrals on Rectangles (Proposition 5.9),

$$
A_{m, n}=F(m+1, n+1) \text { for all }(m, n) \in \mathbb{N}^{2} .
$$

Further, since $a_{k, \ell} \geq 0$ for all $(k, \ell) \in \mathbb{N}^{2}$, it follows from Proposition 7.14 that the double series $\sum \sum_{(k, \ell) \geq(1,1)} a_{k, \ell}$ is convergent if and only if the double sequence $(F(m, n))$ is bounded above, that is, the improper double integral $\iint_{[1, \infty) \times[1, \infty)} f(s, t) d(s, t)$ is convergent, and in this case, the sum of the double series is equal to the value of the improper double integral. Similarly, the double series $\sum \sum_{(k, \ell) \geq(1,1)} a_{k, \ell}$ diverges to $\infty$ if and only if the double sequence $(F(m, n))$ is not bounded above, that is, the improper double integral $\iint_{[1, \infty) \times[1, \infty)} f(s, t) d(s, t)$ diverges to $\infty$.

To relate the convergence of the double series $\sum \sum_{(k, \ell) \geq(1,1)} a_{k, \ell}$ to that of $\sum \sum_{(k, \ell) \geq(1,1)} f(k, \ell)$, observe that since $f$ is monotonically decreasing,

$$
f(k+1, \ell+1) \leq a_{k, \ell} \leq f(k, \ell) \quad \text { for all }(k, \ell) \in \mathbb{N}^{2} .
$$

The first inequality above together with what is shown earlier and the Comparison Test for Double Series (Proposition 7.25) implies that if the improper dou- 
ble integral $\iint_{[1, \infty) \times[1, \infty)} f(s, t) d(s, t)$ is convergent, then $\sum \sum_{(k, \ell) \geq(2,2)} f(k, \ell)$ is convergent, and

$$
\sum_{k=2}^{\infty} \sum_{\ell=2}^{\infty} f(k, \ell)=\sum_{k=1}^{\infty} \sum_{\ell=1}^{\infty} f(k+1, \ell+1) \leq \sum_{k=1}^{\infty} \sum_{\ell=1}^{\infty} a_{k, \ell}=\iint_{[1, \infty) \times[1, \infty)} f(s, t) d(s, t) .
$$

Similarly, if the full double series $\sum \sum_{(k, \ell) \geq(1,1)} f(k, \ell)$ is convergent, then the improper double integral $\iint_{[1, \infty) \times[1, \infty)} f(s, t) d(s, t)$ is convergent, and

$$
\iint_{[1, \infty) \times[1, \infty)} f(s, t) d(s, t)=\sum_{k=1}^{\infty} \sum_{\ell=1}^{\infty} a_{k, \ell} \leq \sum_{k=1}^{\infty} \sum_{\ell=1}^{\infty} f(k, \ell),
$$

and moreover, since $f$ is nonnegative, the series $\sum_{k \geq 1} f(k, 1)$ and $\sum_{\ell \geq 1} f(1, \ell)$ are convergent as well. Since $f$ is also monotonically decreasing, by the Integral Test for functions of one variable (Proposition 9.39 of ACICARA), the improper integrals $\int_{[1, \infty)} f(s, 1) d s$ and $\int_{[1, \infty)} f(1, t) d t$ are convergent, and

$$
\begin{aligned}
\sum_{k=1}^{\infty} \sum_{\ell=1}^{\infty} f(k, \ell)= & f(1,1)+\sum_{k=2}^{\infty} f(k, 1)+\sum_{\ell=2}^{\infty} f(1, \ell)+\sum_{k=2}^{\infty} \sum_{\ell=2}^{\infty} f(k, \ell) \\
\leq & f(1,1)+\int_{1}^{\infty} f(s, 1) d s+\int_{1}^{\infty} f(1, t) d t \\
& +\iint_{[1, \infty) \times[1, \infty)} f(s, t) d(s, t) .
\end{aligned}
$$

Finally, if the improper double integral $\iint_{[1, \infty) \times[1, \infty)} f(s, t) d(s, t)$ diverges to $\infty$, then as seen above, the double series $\sum \sum_{(k, \ell) \geq(1,1)} a_{k, \ell}$ diverges to $\infty$, and so by the Comparison Test for Double Series (Proposition 7.25), the full double series $\sum \sum_{(k, \ell) \geq(1,1)} f(k, \ell)$ diverges to $\infty$. Similarly, if the double series $\sum \sum_{(k, \ell) \geq(2,2)} f(k, \ell)$ diverges to $\infty$, then the improper double integral $\iint_{[1, \infty) \times[1, \infty)} f(s, t) d(s, t)$ diverges to $\infty$.

The above result is not completely analogous to the Integral Test for functions of one variable, which says that if $f:[1, \infty) \rightarrow \mathbb{R}$ is a nonnegative monotonically decreasing function, then the improper integral $\int_{[1, \infty)} f(t) d t$ is convergent if and only if the series $\sum_{k \geq 1} f(k)$ is convergent. (See, for example, $[22$, Proposition 9.39]. In fact, if we define $f, g:[1, \infty) \times[1, \infty) \rightarrow \mathbb{R}$ by

$$
f(s, t):=\left\{\begin{array}{ll}
1 & \text { if } s=1, \\
0 & \text { otherwise }
\end{array} \text { and } g(s, t):= \begin{cases}1 & \text { if } s \in[1,2), \\
0 & \text { otherwise }\end{cases}\right.
$$

then we easily see that the double improper integral $\iint_{[1, \infty) \times[1, \infty)} f(s, t) d(s, t)$ is convergent, but the double series $\sum \sum_{(k, \ell) \geq(1,1)} f(k, \ell)$ diverges to $\infty$, while 
the double series $\sum \sum_{(k, \ell) \geq(2,2)} g(k, \ell)$ is convergent, but the double improper integral $\iint_{[1, \infty) \times[1, \infty)} g(s, t) d(s, t)$ diverges to $\infty$.

Let now $f:[1, \infty) \times[1, \infty) \rightarrow \mathbb{R}$ be a nonnegative monotonically decreasing function. Since $\int_{1}^{n} f(s, 1) d s \leq \sum_{k=1}^{n} f(k, 1)$ and $\int_{1}^{n} f(1, t) d t \leq \sum_{\ell=1}^{n} f(1, \ell)$ for all $n \in \mathbb{N}$, Proposition 7.57 yields the following characterization.

The double series $\sum \sum_{(k, \ell) \geq(1,1)} f(k, \ell)$ is convergent if and only if the improper double integral $\iint_{[1, \infty) \times[1, \infty)} f(s, t) d(s, t)$ as well as the improper integrals $\int_{1}^{\infty} f(s, 1) d s$ and $\int_{1}^{\infty} f(1, t) d t$ are convergent.

The above results are useful in determining whether a double series or an improper double integral is convergent, and in that case, to obtain lower bounds and upper bounds for them. This is illustrated in the example below.

Example 7.58. Let $f(s, t):=1 /(s+t)^{p}$ for $(s, t) \in[1, \infty) \times[1, \infty)$, where $p \in \mathbb{R}$ with $p>0$. Then $f$ is a nonnegative monotonically decreasing function. If $p>2$, then the double series $\sum \sum_{(k, l) \geq(1,1)} f(k, \ell)$ is convergent as we have seen in Example 7.17 (i), and so the improper double integral $\iint_{[1, \infty) \times[1, \infty)} f(s, t) d(s, t)$ is convergent by Proposition 7.57. Conversely, suppose the improper double integral $\iint_{[1, \infty) \times[1, \infty)} f(s, t) d(s, t)$ is convergent. Then the double series $\sum \sum_{(k, l) \geq(2,2)} f(k, \ell)$ is convergent by Proposition 7.57. Hence proceeding as in Example 7.17 (i) and considering the diagonal series $\sum_{j \geq 2} c_{j}$, where $c_{j}:=(j-1) /(j+2)^{p}$, we obtain $p>2$. Thus the improper double integral $\iint_{[1, \infty) \times[1, \infty)} f(s, t) d(s, t)$ is convergent if and only if $p>2$.

Alternatively, we can directly show that $\iint_{[1, \infty) \times[1, \infty)} f(s, t) d(s, t)$ is convergent if and only if $p>2$ as follows. Indeed, let $(x, y) \geq(1,1)$, and let $F(x, y):=\iint_{[1, x] \times[1, y]} d(s, t) /(s+t)^{p}$. Suppose $p \leq 2$. Then

$$
\begin{aligned}
F(x, y) & \geq \iint_{[1, x] \times[1, y]} \frac{d(s, t)}{(s+t)^{2}}=\int_{1}^{x}\left(\int_{1}^{y} \frac{d t}{(s+t)^{2}}\right) d s \\
& =\int_{1}^{x}\left(\frac{1}{s+1}-\frac{1}{s+y}\right) d s=\ln (x+1)-\ln 2-\ln (x+y)+\ln (1+y) \\
& =\ln \frac{(x+1)(y+1)}{x+y}-\ln 2 \geq-\ln \left(\frac{1}{x+1}+\frac{1}{y+1}\right)-\ln 2 .
\end{aligned}
$$

Hence $\iint_{[1, \infty) \times[1, \infty)} d(s, t) /(s+t)^{p}$ diverges to $\infty$. Next, suppose $p>2$. Then

$$
\begin{aligned}
F(x, y) & =\frac{1}{p-1} \int_{1}^{x}\left[\frac{1}{(s+1)^{p-1}}-\frac{1}{(s+y)^{p-1}}\right] d s \\
& =\frac{1}{(p-1)(p-2)}\left[\frac{1}{2^{p-2}}-\frac{1}{(x+1)^{p-2}}-\frac{1}{(1+y)^{p-2}}+\frac{1}{(x+y)^{p-2}}\right] .
\end{aligned}
$$


Hence

$$
\iint_{[1, \infty) \times[1, \infty)} \frac{1}{(s+t)^{p}} d(s, t)=\frac{1}{(p-1)(p-2) 2^{p-2}} \quad \text { if } p>2 .
$$

Now we can obtain an alternative proof of the fact that the double series $\sum \sum_{(k, l) \geq(1,1)} f(k, \ell)$ is convergent if and only if $p>2$ by invoking Proposition 7.57 and by observing that the improper integrals $\int_{1}^{\infty} d s /(s+1)^{p}$ and $\int_{1}^{\infty} d t /(1+t)^{p}$ are convergent when $p>2$.

When $p>2$, Proposition 7.57 allows us to estimate the double sum $\sum \sum_{(k, \ell) \geq(1,1)} 1 /(k+\ell)^{p}$. First we note that if $p>1$, then

$$
\int_{1}^{x} \frac{1}{(s+1)^{p}} d s=\frac{1}{p-1}\left(\frac{1}{2^{p-1}}-\frac{1}{(x+1)^{p-1}}\right) \leq \frac{1}{(p-1) 2^{p-1}} \quad \text { for all } x \geq 1 .
$$

Now

$$
\begin{aligned}
\frac{1}{(p-1)(p-2) 2^{p-2}} & \leq \sum_{(k, \ell) \geq(1,1)} \frac{1}{(k+\ell)^{p}} \\
& \leq \frac{1}{2^{p}}+\frac{1}{(p-1) 2^{p-1}}+\frac{1}{(p-1) 2^{p-1}}+\frac{1}{(p-1)(p-2) 2^{p-2}} \\
& =\frac{p^{2}+p-2}{2^{p}(p-1)(p-2)} .
\end{aligned}
$$

For example,

$$
\frac{1}{4} \leq \sum_{(k, \ell) \geq(1,1)} \frac{1}{(k+\ell)^{3}} \leq \frac{5}{8} \quad \text { and } \quad \frac{1}{24} \leq \sum_{(k, \ell) \geq(1,1)} \sum_{(k+\ell)^{4}} \leq \frac{3}{16}
$$

by letting $p=3$ and $p=4$. Finally, we remark that if we denote by $\zeta(s)$ the sum of the convergent series $\sum_{n \geq 1} 1 / n^{s}$, where $s \in \mathbb{R}$ with $s>1$, then for $p>2$, the sum $\sum_{k=1}^{\infty} \sum_{\ell=1}^{\infty} 1 /(k+\ell)^{p}$ can be expressed as $\zeta(p-1)-\zeta(p)$. Indeed,

$\sum_{k=1}^{\infty} \sum_{\ell=1}^{\infty} \frac{1}{(k+\ell)^{p}}=\sum_{n=2}^{\infty} \sum_{(k, \ell) \geq(1,1)} \frac{1}{n^{p}}=\sum_{n=2}^{\infty} \frac{(n-1)}{n^{p}}=(\zeta(p-1)-1)-(\zeta(p)-1)$

This indicates that it is not easy to find an exact value of the sum of the above double series for $p>2$, even if $p$ is an integer. Indeed, while values of $\zeta(n)$ are known when $n$ is an even positive integer (Theorem 5.6.3 of Hijab's book [30]), values of $\zeta(n)$ when $n$ is an odd integer $>1$ remain mostly a mystery. For instance, it is not known whether or not $\zeta(5)$ is irrational.

p. 438, +10: Change "if and only if" to "if and only if there is" 